\title{
HEVC Video encoder implementation On Texas Instruments Platforms
}

\author{
Nejmeddine Bahri, Abdessamad el Ansari, Mohamed Maazouz, Ali Ahaitouf, Nouri Masmoudi \\ (1) National School of Engineers / LETI Laboratory, University of Sfax-Tunisia \\ (2) RESS Laboratory, Sidi Mohammed Ben Abdellah University, Fez-Morocco \\ (3) LSEA Laboratory, University of Médéa, Algeria \\ nejmeddine.bahri@gmail.com
}

\begin{abstract}
- this paper presents a high efficiency video encoder implementation (HEVC) on two different Texas Instruments (TI) platforms: the BeagleBoard-xM based on ARM processor and the TMS320C6678 DSP. The new features of these processors such as multicore architecture, high frequency processor and low power consumption motivate researchers to develop an embedded HEVC video encoder which could be exploited in several multimedia applications such as High Definition (HD)TV, smart cameras, HD video conference, Ultra HD video surveillance systems. Different operating systems (OS) with different compilers are tested in order to obtain an optimized HEVC encoder implementation. Experimental results show that HEVC encoder DSP-based solution using SYS BIOS real time OS allows saving up to $62 \%$ of encoding time compared to Linux-c6x OS with the same DSP and about $71 \%$ of encoding time compared to BeagleBoard-based solution without inducing any performance degradation in terms of video quality or bitrate.
\end{abstract}

Keywords - HEVC encoder, DSP, ARM processor, RTOS, Cross-Compilation

\section{INTRODUCTION}

Nowadays, the fast evolution of digital cameras technology makes users more consumers of UHD images and videos applications. Facing the huge amount of data transmission, the big memory storing requirement and the transmission bandwidth constraints, video encoding with high compression performance and low computational cost is absolutely required.

The High Efficiency Video Coding HEVC [1] is the new generation of video coding standard. It is developed to overcome the huge amount of UHD video data. The HEVC encoder ensures a high encoding efficiency with respect to the already commercialized H264/AVC encoder. It saves up to $50 \%$ of bitrate with the same visual quality. This efficiency is accompanied by a tremendous computational complexity due to many new features included in this standard.

Develop an embedded solution for this encoder will be an interesting work to be incorporated in several multimedia devices. In fact, smart cameras, mobile phones, smart TV, MP4 players and surveillance systems usually include embedded processors to run video applications.

Currently, the majority of embedded implementations focus on the HEVC decoder which is less complicated and easier for implementation compared to the encoder one [2] [3] [4] and [5]. Regarding the HEVC encoder, there are two implementation aspects: some works focus on software optimizations of different encoder modules such as intra prediction [6], motion estimation [7]. Others are interested in hardware implementations on different platforms such as Field Programmable Gate Array (FPGA) platform and x86 processors with Single Instruction Multiple Data (SIMD) architecture by using openMP library to accelerate encoding speed [8] to [14].

In this paper, hardware implementations of HEVC encoder on two Texas Instruments embedded systems running at the same processor frequency are presented. BeagleBoard-xM platform based on ARM processor and the TMS320C6678 multicore DSP platform are chosen to run the HEVC encoder in order to evaluate and compare their performances. As preliminary work, our implementation is limited to a single core implementation. The motivation of this work is to perform an embedded encoder implementation that could be integrated on several devices such smart cameras, smart TV, UHD surveillance systems etc.

The remainder of this paper is organized as follows: section 2 gives a brief description of the target platforms features. Section 3 presents the HEVC encoder implementation strategies on the BeagleBoard-xM and DSP platforms using Linux operating system. Optimization steps by exploiting a real time operating system are detailed in section 4 . Section 5 concludes the paper and presents some perspectives.

\section{PLATFORMS DESCRIPTION}

\section{A. BeagleBoard-xM Platform}

BeagleBoard-xM [15] is a low cost ARM Cortex A8 board. It is based on the DM3730 processor manufactured by Texas Instruments running at $1 \mathrm{GHz}$ with extra memory@512MB of low-power DDR RAM as shown in Figure 1.

The BeagleBoard-xM is designed specifically to address the Open Source Community. It is characterized by low power consumption and integrates a large amount of On-Chip memory. It supports high-capacity microSD slot and 4-GByte microSD card which makes developers able to boot new operating systems as Angstrom Linux, Ubuntu, and Android without any dependencies on fixed non-volatile memory like NAND flash. Several interfaces are linked to this board such as 10/100 Ethernet for Internet Protocol networks and four high speed USB 2.0 ports used for USB peripherals. It includes a camera port to directly import videos from a connected camera. All these features make the BeagleBoard-xM very interesting solution to run several embedded multimedia applications like image and video processing applications, Robotics etc. 


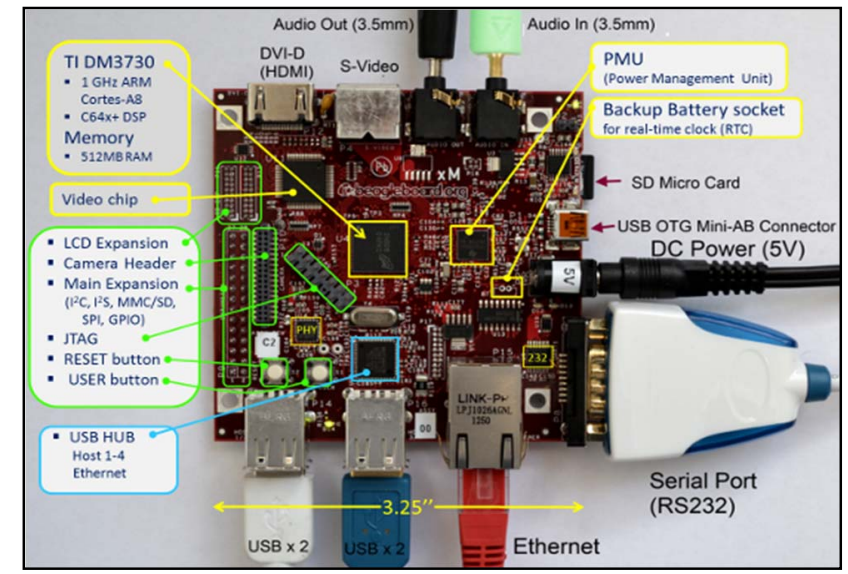

Figure 1. BeagleBoard-xM Platform

\section{B. TMS320C6678 DSP Platform}

DSP processors represent an attractive solution for embedded systems implementations and high performance applications thanks to software flexibility, low power consumption, competitive price tag and time to market. TMS320C6678 DSP [16] belongs to the latest generation of multicore DSPs made by Texas Instrument. It includes eight cores running each @ 1GHz which allows delivering 64000 MIPS performance. It is based on Very Long Instruction Word (VLIW) architecture and uses Single Instruction Multiple Data (SIMD) set instruction. Each C66x core integrates a large amount of on-chip memory. In addition to $32 \mathrm{~KB}$ of L1 program and data cache, it includes $512 \mathrm{~KB}$ of internal memory per core that can be configured as mapped RAM or cache. The platform also integrates 4MB of Multicore Shared Memory and 512MB of DDR3-1333 external memory. This device also includes 64MB of NAND Flash memory to run Linux-c6x Kernel. This platform comes with the TI's Multicore Software Development Kit (MCSDK) for SYS/BIOS Real Time Operating System (RTOS).

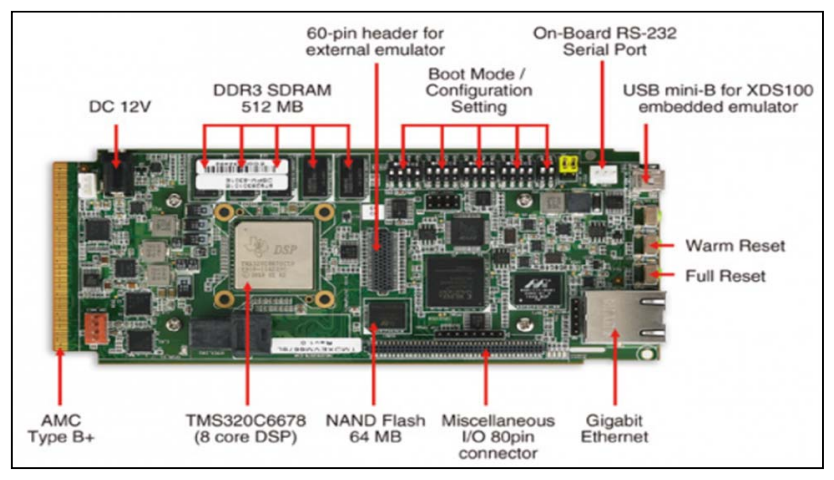

Figure 2. TMS320C6678 DSP Platform

\section{HEVC ENCODER IMPLEMENTATION USING LINUX OPERATING SYSTEMS}

\section{A. HEVC encoder implementation on BeagleBoard-xM}

BeagleBoard-xM platform is based on DM3730 processor. This device supports high-level operating systems such as Windows CE, Linux, ubuntu and QNX etc. This platform comes with a $4 \mathrm{~GB}$ microSD card that supports an Angstrom
Linux validation image. To perform a functional HEVC video encoding application on the BeagleBoard-xM platform, we are processed as follows:

- A cross compile toolchain for ARM BeagleBoard-xM architecture is installed on the host machine in order to compile and build the HEVC encoder software.

- An Angstrom Linux kernel is installed in the SD card of the BeagleBoard-xM.

- The HEVC encoder is then compiled with the ARM cross compiler. An executable cross compiled binary output is generated. It is then copied with the input videos and the encoder configuration file ".cfg" into the SD card.

- The BeagleBoard-xM, which is connected with the host machine via the serial port RS232, boots on Linux from this SD card.

- minicom or picocom tool, which are a text-based serial port communications programs could be used to ensure communication between the host and the Beagleboard platform. Thus, execution of the binary executable can be started.

\section{B. HEVC encoder implementation on TMS320C6678 DSP platform}

As a preliminary work, only a mono-core HEVC encoder implementation is presented. For future work, a multicore implementation will be performed.

The HEVC software cannot be directly executed on the DSP using C6000 compiler with SYS/BIOS, the Real time operating system (RTOS) for DSP processor. The HEVC software includes several functions and data structures that are not compatible with DSP development frameworks. Thus, several changes should be performed in order to obtain a DSP functional version as Pescador's implementation [5].

To avoid these modifications and easily obtain HEVC DSPbased solution, increasing the level of abstraction by exploiting a Linux gec compiler instead of the standard C6000 SYS/BIOS compiler could be an efficient solution. In fact, TMS320C6678 includes a $64 \mathrm{MB}$ NAND Flash memory that can support a Linux operating system. So, the key idea is exploiting Linux instead of SYS/BIOS and a $\mu$ clinux-c $6 x$ cross compiler instead of the standard C6000 compiler to compile and build the HEVC software.

The implementation steps of the HEVC encoder on the TMS320CC6678 DSP using a Linux OS are summarized as follows:

- Linux-c6x kernel, which is an embedded Linux dedicated for C6000 DSP family, is installed and loaded into the Nand Flash memory [17].

- The HEVC encoder software is build using $\mu$ clinux-c6x cross compiler.

- To ensure that the DSP exploits its entire memory in processing and loading Linux files system, the HEVC binary executable, the input video sequences and the 
encoder configuration file ".cfg" are kept on the host machine in a shared folder. The DSP will access to this shared folder with the Network File System (NFS) protocol [18].

- The EVM switches are configured according to the NAND flash boot mode.

- The DSP and the host machine are interconnected via both the Ethernet link using a static IP addresses and the serial RS232 interface to ensure between them.

- Another folder is created in the DSP file system. It will be used as the mount point. NFS command is then used to access to the shared folder on the host machine where the HEVC binary image and the video sequences have been saved. The DSP can now access to the HEVC executable binary code and the application could be executed without modifying any function or data structure or take into account data allocations into the DSP memory.

\section{Experimental results with Linux Operating Systems}

To evaluate our implementations performances, the HEVC encoder reference software (HM 12.1) [19] is executed on the both platforms that have the same processor frequency. In fact, BeagleBoard-xM ARM processor and the C6678 DSP core are both running at $1 \mathrm{GHz}$.

Experimental conditions are similar to common test conditions [20] in HEVC standard. The largest coding unit (LCU) is fixed to $64 \times 64$ with a maximum depth level equal to 4. The entropy coder is CABAC (Context Adaptive Binary Arithmetic Coding). Different video sequences recommended by the JCT-VC [21] are used for tests. The number of encoded frame for each video sequence is 100 . All the simulations are evaluated with four QPs (Quantification Parameter) 22, 27, 32 and 37. As preliminary tests, only Class D (416x240) video resolution and "ALL INTRA" configuration are used to evaluate the performance of these implementations.

Table I presents the average encoding time (seconds) for one frame using Linux Operating System on BeagleBoard-xM and DSP TMS320C6678 platforms. Regarding quality degradation or bitrate increase, these two criteria have not changed since we have not made any modifications in the HEVC software. Only encoding time is taken into account to evaluate our implementations performance.

Time saving is also computed as follows:

Time saving $(\%)=\frac{\text { Enc time on Beagle }- \text { Enc Time on DSP }}{\text { Enc time on Beagle }}$

Experiments confirm that the HEVC video encoder represents a high computational complexity. An important execution time is required to encode one frame even for low video resolution. Experimental results show that our single core DSP implementation can save about $20 \%$ in average of encoding time compared to BeagleBoard-xM. This time saving may return to the C6678 external memory bandwidth where most of required encoding data are allocated compared to that of BeagleBoard-xM. In fact, C6678 includes a 64-bits DDR-3 external memory interface (EMIF) running at $1333 \mathrm{MHz}$ whereas DDR's BeagleBoard is a 32 bits running at $166 \mathrm{MHz}$. This drastically affects the encoding time since the HEVC encoder includes an important number of Load/Store data instructions. Moreover, the C6678 includes a large amount of on-Chip memory $(512 \mathrm{~KB}$ of internal memory per core and $4 \mathrm{MB}$ of Multicore Shared Memory) characterized by high bandwidth which may reduce memory latency and speed up the encoding run-time.

TABLE I. AVERAGE ENCODING TIME FOR CLASS D (416X240) VIDEO SEQUENCES

\begin{tabular}{|c|c|c|c|c|}
\hline $\begin{array}{c}\text { Class D } \\
(416 \times 240) \\
\text { video } \\
\text { sequences }\end{array}$ & QP & $\begin{array}{l}\text { Average encoding } \\
\text { time for one frame } \\
\text { on BeagleBoard (s) }\end{array}$ & $\begin{array}{l}\text { Average encoding } \\
\text { time for one frame } \\
\text { on C6678 DSP (s) }\end{array}$ & $\begin{array}{c}\text { Time } \\
\text { saving } \\
(\%)\end{array}$ \\
\hline \multirow{4}{*}{$\begin{array}{l}\text { Basketball } \\
\text { Pass }\end{array}$} & 22 & 23,07 & 18,23 & 20,98 \\
\hline & 27 & 20,24 & 16,09 & 20,50 \\
\hline & 32 & 17,75 & 14,57 & 17,92 \\
\hline & 37 & 15,90 & 13,35 & 16,04 \\
\hline \multirow{4}{*}{ BQSquare } & 22 & 29,59 & 22,72 & 23,22 \\
\hline & 27 & 26,15 & 20,05 & 23,33 \\
\hline & 32 & 22,72 & 17,97 & 20,91 \\
\hline & 37 & 20,10 & 16,12 & 19,80 \\
\hline \multirow{4}{*}{$\begin{array}{l}\text { Blowing } \\
\text { Bubbles }\end{array}$} & 22 & 28,77 & 22,25 & 22,66 \\
\hline & 27 & 24,79 & 19,18 & 22,63 \\
\hline & 32 & 21,02 & 16,71 & 20,50 \\
\hline & 37 & 17,88 & 14,62 & 18,23 \\
\hline \multirow{4}{*}{$\begin{array}{l}\text { Race } \\
\text { Horses }\end{array}$} & 22 & 26,58 & 20,48 & 22,95 \\
\hline & 27 & 23,54 & 18,25 & 22,47 \\
\hline & 32 & 20,28 & 16,71 & 17,60 \\
\hline & 37 & 17,88 & 14,33 & 19,85 \\
\hline $\begin{array}{l}\text { Average } \\
\text { time }\end{array}$ & & 22,27 & 17,60 & 20,60 \\
\hline
\end{tabular}

Based on these results and in order to speed up the encoding time, we have decided to keep working with the DSP platform and moving to use the SYS BIOS real time operating system with the C6000 compiler and the Integrated Development Environment (IDE) Code Composer Studio v5 [22].

\section{HEVC ENCODER IMPLEMENTATION USING REAL TIME OPERATING SYSTEM}

\section{A. HEVC implementation details on DSP platform}

To obtain a DSP-based functional version using C6000 compiler, some modifications have been done in the $\mathrm{C}++$ HEVC reference software in order to be compatible with DSP frameworks. The most important changes in the original code were:

- Use the string library instead of the memory library.

- Redefine the function "find" included in the class TComIterator to adapt the input parameters.

- Include math library and use abs intrinsic function that calculates the absolute value of a variable.

- Redefine some $\mathrm{C}++$ classes to adapt some variables unsupported by the DSP C++ compiler. 
- Define the type "bool" in the encoder in order to be recognized by the C6000 compiler.

- Development of the function strdup, that is unavailable in the DSP development environment.

- Replace the functions used to measure the performance with PC timers by the appropriate functions that use DSP timers.

- Configure the compiler output format to ELF (Executable and Linkable Format) instead of COFF (Common Object File Format), needed to support some features of the $\mathrm{C}++$ code.

- Check the compiler option "Support $\mathrm{C}++$ run-time type information -rtti" used to determine the type of a variable during the execution of the program.

\section{B. Experimental results with SYS BIOS Real Time Operating System}

By applying the changes indicated above, we have succeeded to obtain a DSP-based functional version with SYS BIOS operating system and C6000 compiler.

To compare between the two operating systems: Linux-C6x and SYS BIOS, the same test conditions and the same video sequences are used. Table II presents the average encoding time for class D $(416 \times 240)$ video sequences with different QP values.

TABLE II. HEVC ENCODING TIME ON DSP PLATFORM USING LINUX-C6X AND SYS BIOS FOR CLASS D VIDEO SEQUENCES

\begin{tabular}{|c|c|c|c|c|}
\hline $\begin{array}{c}\text { Class D } \\
(416 \times 240) \\
\text { video } \\
\text { sequences }\end{array}$ & QP & $\begin{array}{l}\text { Average encoding } \\
\text { time for one frame } \\
\text { on C6678 using } \\
\text { Linux-c6x (s) }\end{array}$ & $\begin{array}{l}\text { Average encoding } \\
\text { time for one frame } \\
\text { on C6678 using } \\
\text { SYS BIOS (s) }\end{array}$ & $\begin{array}{c}\text { Time } \\
\text { saving } \\
(\%)\end{array}$ \\
\hline \multirow{4}{*}{$\begin{array}{l}\text { Basketball } \\
\text { Pass }\end{array}$} & 22 & 18,23 & 13,70 & 24,85 \\
\hline & 27 & 16,09 & 11,83 & 26,48 \\
\hline & 32 & 14,57 & 10,39 & 28,69 \\
\hline & 37 & 13,35 & 9,32 & 30,19 \\
\hline \multirow{4}{*}{ BQSquare } & 22 & 22,72 & 16,74 & 26,32 \\
\hline & 27 & 20,05 & 14,61 & 27,13 \\
\hline & 32 & 17,97 & 12,71 & 29,27 \\
\hline & 37 & 16,12 & 11,32 & 29,78 \\
\hline \multirow{4}{*}{$\begin{array}{l}\text { Blowing } \\
\text { Bubbles }\end{array}$} & 22 & 22,25 & 16,53 & 25,71 \\
\hline & 27 & 19,18 & 14,03 & 26,85 \\
\hline & 32 & 16,71 & 11,90 & 28,79 \\
\hline & 37 & 14,62 & 10,27 & 29,75 \\
\hline \multirow{4}{*}{$\begin{array}{l}\text { Race } \\
\text { Horses }\end{array}$} & 22 & 20,48 & 15,45 & 24,56 \\
\hline & 27 & 18,25 & 13,55 & 25,75 \\
\hline & 32 & 16,71 & 11,77 & 29,56 \\
\hline & 37 & 14,33 & 10,26 & 28,40 \\
\hline $\begin{array}{l}\text { Average } \\
\text { time }\end{array}$ & & 17,60 & 12,77 & 27.43 \\
\hline
\end{tabular}

Experimental results show that the encoding time is improved by about 27\% when using SYS BIOS and C6000 compiler compared to the implementation version using LinuxC6x and $\mu$ clinux-c6x compiler. Hence, the average Class D encoding time is decreased from 17.6 seconds with Linux OS to 12.77 seconds with SYS BIOS.
This enhancement returns to that $\mathrm{C} 6000$ compiler used with SYS BIOS outperforms the uClinux-C6x compiler used with Linux-C6x OS in terms of generating a well optimized assembly code that takes into account the internal architecture of the TMS320C6678 DSP platform.

\section{System optimizations of HEVC implementation with SYS BIOS Real Time Operating System}

Code Composer studio (CCS) environment gives the possibility to apply various optimizations either from build options project or from SYS BIOS configuration file based on the XDCtools [23]. This last one is a software product installed with CCS that contains all of the tools necessary to create, test, deploy, install, and use RTSC components [24] (Real Time Software component called packages) of SYS BIOS and to configure the application.

\section{1) Configuration of program sections Map}

During the first implementation of HEVC encoder on the C6678 DSP with SYS BIOS, all program memory sections have been allocated in the external DDR3 memory to avoid memory allocation problems since its size (512 MByte) is sufficient to support all data allocations of HEVC encoder. To optimize our implementation, we have decided to use the local internal L2RAM memory (512 KByte of size) and the shared internal MSMCSRAM memory (4 MByte of size) to allocate some program sections in order to get a faster data access compared to the external DDR3 memory.

Therefore, as shown in Table III, the ".text" section which presents the segment that contains the executable program code is configured in the shared MSMCSRAM memory (4 MByte) as its size is about 1 MByte. The "heap" segment which concerns dynamic allocation, is kept in the external DDR3 memory to support large memory allocations. Other sections, such as ".stack" which includes local variables, ".bss" for global variables, ".cinit" for initial values are allocated in the internal L2RAM memory since their sizes do not exceed 250 KByte.

TABLE III. PROGRAM SECTIONS MAP CONFIGURATION

\begin{tabular}{|l|l|}
\hline Program.sectMap ["heap"] & $=$ "DDR3"; \\
\hline Program.sectMap [".text"] & $=$ "MSMCSRAM"; \\
\hline Program.sectMap [".far"] & $=$ "L2SRAM"; \\
\hline Program.sectMap [".cinit"] & $=$ "L2SRAM"; \\
\hline Program.sectMap [".rodata"] & $=$ "L2SRAM"; \\
\hline Program.sectMap [".switch"] & $=$ "L2SRAM"; \\
\hline Program.sectMap [".args"] & $=$ "L2SRAM"; \\
\hline Program.sectMap [".bss"] & $=$ "L2SRAM"; \\
\hline Program.sectMap [".neardata"] & $=$ "L2SRAM"; \\
\hline Program.sectMap [".stack"] & $=$ "L2SRAM"; \\
\hline Program.sectMap [".data"] & $=$ "L2SRAM"; \\
\hline Program.sectMap [".cio"] & $=$ "L2SRAM"; \\
\hline Program.sectMap [".vecs"] & $=$ "L2SRAM"; \\
\hline
\end{tabular}

2) Cache memory activation

In addition to program sections Map configuration, SYS BIOS gives the possibility to configure the target platform via the RTSC tool. Hence, to optimize our implementation, we have configured a part of L2RAM memory as a cache to speed up the data processing. In the first implementation, the whole L2 memory was configured as data memory. Since the L2RAM 
size is 512 KByte and knowing that the memory sections that have been allocated in don't exceed the $256 \mathrm{KByte}$, in this case the L2 cache size has been set to $256 \mathrm{~KB}$. The L1D memory (Data memory Level 1) and L1P (Program memory Level 1) are always configured as $32 \mathrm{Kbyte}$ of cache even for the first implementation with SYS BIOS.

\section{3) Project Build options optimizations}

In order to generate a well optimized code level parallelism and software pipeline, the CCS environment provides the possibility to set the project build options that the C6000 compiler must take into account when compiling the code and generating the executable file. Consequently, the build options of our HEVC encoder project are fixed as follows:

- Build configuration mode: Release

- Optimization level (--opt_level,O): 3

- Optimize for code size (--opt_for_space,-ms) : 3

- Optimize for speed (--opt_for_speed, -mf): 5

- Inline function called once --single_inline: checked

D. Experimental results with system optimizations using the SYS BIOS real time operating system

The HEVC encoder is executed on the C6678 DSP after applying all the proposed system optimizations. The same test conditions are used in this implementation. Table IV shows a comparison between the experimental results of the nonoptimized version and the optimized one with SYS BIOS real time operating system.

TABLE IV. HEVC ENCODING PERFORMANCE ON C6678 DSP USING SYS BIOS WITH SYSTEM OPTIMIZATIONS

\begin{tabular}{|c|c|c|c|c|}
\hline $\begin{array}{c}\text { Class D } \\
(416 \times 240) \\
\text { video } \\
\text { sequences }\end{array}$ & QP & $\begin{array}{l}\text { Encoding time for } \\
\text { one frame on } \\
\text { C6678 DSP using } \\
\text { SYS BIOS } \\
\text { without system } \\
\text { optimizations (s) }\end{array}$ & $\begin{array}{l}\text { Encoding time for } \\
\text { one frame on } \\
\text { C6678 DSP using } \\
\text { SYS BIOS with } \\
\text { system } \\
\text { optimizations (s) }\end{array}$ & $\begin{array}{c}\text { Time } \\
\text { saving } \\
(\%)\end{array}$ \\
\hline \multirow{4}{*}{$\begin{array}{l}\text { Basketball } \\
\text { Pass }\end{array}$} & 22 & 13,70 & 6,97 & 49,12 \\
\hline & 27 & 11,83 & 6,07 & 48,69 \\
\hline & 32 & 10,39 & 5,35 & 48,51 \\
\hline & 37 & 9,32 & 4,81 & 48,39 \\
\hline \multirow{4}{*}{ BQSquare } & 22 & 16,74 & 8,46 & 49,46 \\
\hline & 27 & 14,61 & 7,41 & 49,28 \\
\hline & 32 & 12,71 & 6,51 & 48,78 \\
\hline & 37 & 11,32 & 5,80 & 48,76 \\
\hline \multirow{4}{*}{$\begin{array}{l}\text { Blowing } \\
\text { Bubbles }\end{array}$} & 22 & 16,53 & 8,26 & 50,03 \\
\hline & 27 & 14,03 & 7,12 & 49,25 \\
\hline & 32 & 11,90 & 6,10 & 48,74 \\
\hline & 37 & 10,27 & 5,30 & 48,39 \\
\hline \multirow{4}{*}{$\begin{array}{l}\text { Race } \\
\text { Horses }\end{array}$} & 22 & 15,45 & 7,86 & 49,13 \\
\hline & 27 & 13,55 & 6,95 & 48,71 \\
\hline & 32 & 11,77 & 6,06 & 48,51 \\
\hline & 37 & 10,26 & 5,30 & 48,34 \\
\hline $\begin{array}{l}\text { Average } \\
\text { time }\end{array}$ & & 12,77 & 6,52 & 48,88 \\
\hline
\end{tabular}

Experimental results show that the applied optimizations have ensured up to $48 \%$ of encoding time saving compared to the non-optimized version. In fact, the encoding time of a Class $\mathrm{D}$ image is decreased from 12.7 seconds to 6.52 seconds in average.

As result, our optimized implementation of the HEVC encoder on the C6678 DSP using SYS BIOS real time operating system allows saving up to $71 \%$ of encoding time compared to the implementation on the BeagleBoard-xM. In fact, the encoding time is decreased from 22.27 seconds on BeagleBoard-xM to 6.52 seconds on the C6678 DSP. Moreover, the use of SYS BIOS and C6000 compiler instead of Linux-c6x has saved up to $62 \%$ of encoding time which is decreased from 17.60 seconds with Linux-c6x to 6.52 seconds with SYS BIOS. Finally, we can consider this work as an important step to develop an embedded HEVC encoder despite the high complexity of this standard. This work will be the starting point for future researches. We have succeeded to obtain a functional HEVC encoder embedded on two different Texas Instruments processors without inducing any encoding degradation in terms of video quality or bitrate.

In order to improve the encoding time and reduce the computational complexity of the HEVC encoder, several optimizations could be proposed such as proposing fast coding algorithms and more optimized implementations exploiting the fully platform architecture. It is also possible to use pragmas instructions (MUST ITERATE, UNROLL, INLINE etc) to optimize loops and functions. Moreover, a low-level assembly language programming of some functions such as SAD (Sum of Absolute Difference), SSE (Sum of Square Error), HAD (Transform HADAMARD) could also significantly improve the encoding speed as these functions are several times called for each Coding Unit (CU). Finally, exploiting the multicore architecture of the C6678 DSP and moving to a parallel implementation could be an interesting solution to accelerate the encoding process.

\section{CONCLUSION}

This paper proposed a HEVC video encoder implementation on two different platforms: BeagleBoard-xM based on ARM processor and the TMS320C6678 DSP, running both at the same processor frequency $1 \mathrm{GHz}$. Different operating systems with different compilers have been tested in order to obtain an optimized implementation of this encoder. We have compared between the use of Linux operating systems and SYS BIOS real time operating system in terms of encoding time. Experimental results have shown that HEVC encoder DSP-based solution using SYS BIOS allows saving up to $62 \%$ of encoding time compared to the same DSP solution when using Linux-c6x operating system. Moreover, the DSP-based solution with SYS BIOS has saved about $71 \%$ of encoding time compared to BeagleBoard-based solution. Our implementations have not induced any performance degradation in terms of video quality or bitrate. Future work will be focused on the parallel implementation of the HEVC encoder on the multicore TMS3206678 DSP including eight C66x cores. This may give the possibility to reach real-time encoding when combined with architectural, software, and algorithmic optimizations. 


\section{REFERENCES}

[1] B. Bross, W.J. Han, J.R. Ohm, G.J. Sullivan, Y.K. Wang and T.Wiegand. JCTVC-L1003_v30: "High Efficiency Video Coding (HEVC) text specification draft 10 (for FDIS \& Consent)". March 2013.

[2] Chi, C.; Alvarez-Mesa, M.; Lucas, J.; Juurlink, B. \& Schierl, T.Parallel HEVC Decoding on Multi- and Many-core Architectures Journal of Signal Processing Systems, Springer US, 2013, 71, 247-260

[3] Chavarrias, M.; Pescador, F.; Garrido, M.; Juárez, E.; Raulet, M., "A DSP-Based HEVC decoder implementation using an actor language dataflow model," Consumer Electronics, IEEE Transactions on , vol.59, no.4, pp.839,847, November 2013

[4] Pescador, F.; Chavarrias, M.; Garrido, M.J.; Juarez, E.; Sanz, C., "Complexity analysis of an HEVC decoder based on a digital signal processor," Consumer Electronics, IEEE Transactions on, vol.59, no.2, pp.391,399, May 2013.

[5] Pescador, F.; Cano, J.P.; Garrido, M.J.; Juarez, E.; Raulet, M., "A DSP HEVC decoder implementation based on OpenHEVC," Consume Electronics (ICCE), 2014 IEEE International Conference, pp.61,62, 1013 Jan. 2014.

[6] Sangkwon Na; Wonjae Lee; Kiwon Yoo, "Edge-based fast mode decision algorithm for intra prediction in HEVC," Consumer Electronics (ICCE), 2014 IEEE International Conference, pp.11,14, 10-13 Jan. 2014.

[7] Belghith, F.; Kibeya, H.; Loukil, H.; Ben Ayed, M. \& Masmoudi, N. A new fast motion estimation algorithm using fast mode decision for highefficiency video coding standard Journal of Real-Time Image Processing, Springer Berlin Heidelberg, 2014, 1-17

[8] Miyazawa, K.; Sakate, H.; Sekiguchi, S.-I.; Motoyama, N.; Sugito, Y.; Iguchi, K.; Ichigaya, A.; Sakaida, S.-I., "Real-time hardware implementation of HEVC video encoder for 1080p HD video," Picture Coding Symposium (PCS), 2013 , vol., no., pp.225,228, 8-11 Dec. 2013.

[9] Iguchi, K.; Ichigaya, A.; Sugito, Y.; Sakaida, S.; Shishikui, Y.; Hiwasa, N.; Sakate, H.; Motoyama, N., "HEVC encoder for Super HiVision," Consumer Electronics (ICCE), 2014 IEEE International Conference on , vol., no., pp.57,58, 10-13 Jan. 2014,

[10] Jia Zhu; Zhenyu Liu; Dongsheng Wang, "Fully pipelined DCT/IDCT/Hadamard unified transform architecture for HEVC Codec," Circuits and Systems (ISCAS), 2013 IEEE International Symposium on, vol., no., pp.677,680, 19-23 May 2013.

[11] Bin Peng; Dandan Ding; Xingguo Zhu; Lu Yu, "A hardware CABAC encoder for HEVC," Circuits and Systems (ISCAS), 2013 IEEE International Symposium on , vol., no., pp.1372,1375, 19-23 May 2013 doi: 10.1109/ISCAS.2013.6572110
[12] Rediess, F.; Agostini, L.; Cristani, C.; Dall'Oglio, P.; Porto, M., "High throughput hardware design for the Adaptive Loop Filter of the emerging HEVC video coding," Integrated Circuits and Systems Design (SBCCI), 2012 25th Symposium on pp.1,5, Aug. 30 2012-Sept.

[13] Ahn, Y.-J.; Hwang, T.-J.; Sim, D.-G. \& Han, W.-J. Implementation of fast HEVC encoder based on SIMD and data-level parallelism EURASIP Journal on Image and Video Processing, 2014, 2014, 16

[14] Keji Chen; Yizhou Duan; Leju Yan; Jun Sun; Zongming Guo, "Efficient SIMD optimization of HEVC encoder over X86 processors," Signal \& Information Processing Association Annual Summit and Conference (APSIPA ASC), 2012 Asia-Pacific , vol., no., pp.1,4, 3-6 Dec. 2012

[15] BeagleBoard-xM Rev C system Reference Manual, online available: http://beagleboard.org/static/BBxMSRM_latest.pdf

[16] TMS320C6678 Multicore Fixed and Floating-Point Digital Signal Processor Data Manual, Literature Number: SPRS691D April 2013 , online available: http://www.mouser.com/ds/2/405/sprs691d-256638.pdf

[17] Linux-c6x-2.0-Beta2 release, online available: http://linuxc6x.org/wiki/index.php/Linux-c6x_2.0-Beta2_Release

[18] J. Postel and J. Reynolds. File Transfer Protocol. Internet STD 9, RFC 959, Oct. 1985.

[19] K.McCann, B.Bross, W.-J.Han, I.K.Kim, K.Sugimoto, G. J. Sullivan: High Efficiency Video Coding (HEVC) Test Model 12 (HM 12) Encoder Description. In: Proceeding of the 14th JCT-VC Meeting, Vienna (2013).

[20] Bossen, F, JCT-VC-L1100: Common test conditions and software reference configuration. In: Proceeding of the 12th JCT-VC Meeting, Geneva (2013).

[21] Antonio J. Daz-Honrubia, Jos Luis Martnez and Pedro Cuenca, "HEVC: A Review, Trends and Challenges", In: 2nd Workshop on Multimedia Data Coding and Transmission, September 2011.

[22] Code Composer Studio (CCS) Integrated Development Environment (IDE), online available: http://www.ti.com/tool/CCSTUDIO

[23] XDCtools User's guide, online available: http://rtsc.eclipse.org/docstip/XDCtools User\% $\% 27 \mathrm{~s}$ Guide\#Getting started

[24] Real-Time Software Components (RTSC), online available: https://wiki.eclipse.org/DSDP/RTSC 